\title{
Epithelial cell turnover in relation to ongoing damage of the gastric mucosa in patients with early gastric cancer: increase of cell proliferation in paramalignant lesions
}

\author{
Akiko Shiotani ${ }^{1}$, Hiroyasu Iishi ${ }^{2}$, Shingo Ishiguro ${ }^{2}$, Masaharu Tatsuta ${ }^{2}$, Yukinori NakaE ${ }^{3}$, \\ and JuAnita L. Merchant ${ }^{4}$ \\ ${ }^{1}$ Health Administration Center, Wakayama University, 930 Sakaedani, Wakayama 640-8510, Japan \\ ${ }^{2}$ Department of Gastrointestinal Oncology, Osaka Medical Center for Cancer and Cardiovascular Diseases, Osaka, Japan \\ ${ }^{3}$ Aishinkai Nakae Hospital, Wakayama, Japan \\ ${ }^{4}$ Departments of Internal Medicine and Molecular and integrative Physiology, University of Michigan, Ann Arbor, MI, USA
}

Background. Gastric cancer is typically an end result of Helicobacter pylori-associated chronic gastritis. The pathogenesis is thought to involve effects on gastric mucosal epithelial cell turnover. In this study, we aimed to compare apoptosis and proliferation in the noncancer-containing mucosa of $H$. pylori-positive patients with early gastric cancer with these phenomena in H. pylori-positive controls. Methods. Two specimens each were obtained from the greater and lesser curvatures of the corpus and from the greater curvature of the antrum. The histopathological grading used was the updated Sydney System. Apoptotic epithelial cells were detected using the terminal deoxy nucleotidyl transferase-mediated deoxy-uridine triphosphate (dUTP) biotin nick-end labeling (TUNEL) method. The expression of Ki 67 was evaluated by immunostaining. Results. Forty-five H. pylori-positive patients with endoscopic mucosal resection for early gastric cancer and $52 \mathrm{H}$. pylori-positive controls were studied. Gastric cancer was associated with a higher frequency of incomplete intestinal metaplasia (IM; odds ratio [OR], 19.1; 95\% confidence interval [CI], 6.9-53.2; $P<0.001)$. The apoptotic index (AI) in the greater curvature of the corpus and the proliferation index (PI) in each part were significantly higher in cancer patients than in the control group. The median PI in the antrum was significantly higher in the incomplete IM group than that in the complete IM group (17.6 vs 12.6; $P=0.009)$. The PI and the AI in the greater curvature of the corpus correlated with the activity score, and the PI correlated with the IM score. Conclusions. In the cancer patients, $H$. pylori-induced gastritis was associated with increased cell proliferation and apoptosis compared with mucosal findings in the controls. IM

Received: June 8, 2004 / Accepted: December 24, 2004 Reprint requests to: A. Shiotani seems to be one of the most important factors affecting cell proliferation and may be one of the components of carcinogenesis that results in proliferation-dominant cell kinetics.

Key words: intestinal metaplasia, gastric cancer, proliferation, apoptosis, Helicobacter pylori

\section{Introduction}

The evolution of Helicobacter pylori-associated gastric cancer begins with superficial gastritis that progresses to chronic gastritis, atrophy with islands of intestinal metaplasia (IM), development of dysplasia, and, finally, frank carcinoma. ${ }^{1-4}$ There is strong evidence that $H$. pylori infection alters the kinetic pattern of the gastric glandular epithelium. Effects of the infection on gastric mucosal epithelial cell turnover are thought to contribute to the process of carcinogenesis, with disturbances in the balance between cellular proliferation and apoptosis predisposing to either cell loss with mucosal damage or cell accumulation and cancer development.

Recent studies suggest that $H$. pylori infection induces cell apoptosis, a genetically regulated form of programmed cell death. ${ }^{5-9}$ Moss et al. ${ }^{9}$ reported that gastric epithelial apoptosis was enhanced in duodenal ulcer patients with $H$. pylori infection and that the increased number of apoptotic cells returned to normal after $H$. pylori eradication. On the other hand, other studies have reported that $H$. pylori infection is not associated with significant changes in gastric epithelial apoptosis, because there is wide variability in the apoptotic indices of both $H$. pylori-positive and -negative patients. ${ }^{10}$

The aim of this study was to compare apoptosis and proliferation in the non-cancer-containing mucosa of patients with early gastric cancer with these phenomena 
in $H$. pylori-positive controls. We also assessed the relationship between cell turnover and histological findings.

\section{Methods}

This was a case-control study of patients with a history of endoscopic mucosal resection (EMR) for early gastric cancer. The study was performed in Japan, at the Osaka Medical Center for Cancer and Cardiovascular Diseases and at Aishinkai Nakae Hospital. Patients were enrolled for the study between November 2001 and June 2003.

\section{Patients}

H. pylori-positive patients with a medical history of EMR for early stage, noncardiac intestinal type of gastric cancer without lymph node metastasis, and $H$. pylori-positive, age- and sex-matched control patients were eligible for participation. Patients were excluded if they had had eradication therapy for H. pylori infection; used anti-secretory drugs or nonsteroidal antiinflammatory drugs (NSAIDs); or had hemorrhagic diseases, insulin-dependent diabetes mellitus, cirrhosis, or renal failure. Demographic data collected at study entry included age, sex, smoking habits, alcohol consumption, and drug treatments, including the use of anti-secretory drugs. Drinking and smoking were defined as regular intake when consumption was more than $35 \mathrm{~g}$ of ethanol or 5 cigarettes or more per day, respectively. The study was approved by the Osaka Medical Center for Cancer and Cardiovascular Diseases Ethics Committee, and informed consent was obtained from each patient.

\section{Histopathological grading}

Endoscopies were performed by experienced endoscopists after the patients had had a 12-h fast. Two specimens each were obtained from the greater curvature of the antrum and the greater and lesser curvature of the corpus. The specimens were stained with hematoxylin and eosin, and additionally stained with Giemsa to score $H$. pylori density. A visual analogue scale, graded from 0 (absent/normal) to 3 (maximal intensity) was used for the following parameters: activity of gastritis (granulocytic infiltration), inflammation (lymphocytic and plasma cells infiltration), glandular atrophy, IM, and $H$. pylori density, according to the updated Sydney classification. ${ }^{11}$ Well-oriented tissue sections with gastric pits and glands cut longitudinally and visible along the entire isthmus zone were selected for quantitative analysis. Histopathological grading was deter- mined twice for each slide, by a single pathologist who was blinded to the previous histological scores and other experimental results. Average histological scores were used to analyze the data.

\section{Diagnosis of $\mathrm{H}$. pylori infection}

Venous blood samples were analyzed for specific IgG H. pylori antibodies with an enzyme-linked immunosorbent assay (ELISA) kit, using the E plate test (Eiken Kagaku, Tokyo, Japan). Patients were considered to be infected with $H$. pylori if the serum test was positive or if chronic gastritis/atrophy with $H$. pylori was histopathologicaly present.

\section{Subtyping IM}

Samples were also stained with Alcian blue (AB)/high iron diamine (HID) to categorize IM as complete type (type I) or incomplete type (types II and III). The slides were immersed in HID solution for $20 \mathrm{~h}$ at room temperature, then rinsed with deionized water and stained with $1 \% \mathrm{AB}(\mathrm{pH} 2.5)$ for $2 \mathrm{~min}$.

\section{Immunohistochemistry}

Three adjacent sections, 4- $\mu \mathrm{m}$-thick, were cut onto each of three polylysine-coated glass slides. The sections were deparaffinized, washed in phosphate-buffered saline (PBS), and autoclaved for $10 \mathrm{~min}$ in sodium citrate buffer for antigenic retrieval. For immunostaining, sections were incubated with mouse monoclonal anti-human Ki-67 (DAKO; Carpinteria, CA, USA) at a dilution of $1: 100$ at room temperature for $1 \mathrm{~h}$. After a washing in PBS, the sections were incubated for $1 \mathrm{~h}$ with an anti-mouse immunoglobulin conjugated to horseradish peroxidase dextran-labeled polymer, using an EnVision peroxidase mouse system (DAKO). The sections were washed in PBS, stained in $0.05 \%$ diaminobenzidine hydrochrolide (DAB) solution for 5 min, then counterstained with hematocxylin. After dehydration with xylene, the sections were mounted under a glass coverslip. More than 500 cells along the proliferation zone were counted, and Ki-67-positive cells were expressed as a percentage of the total number of cells, as the proliferative index (PI).

\section{Detection of apoptotic cells}

Apoptotic cells were detected by the terminal deoxynucleotidyl transferase-mediated deoxyuridine triphosphate (dUTP) biotin nick-end labeling (TUNEL) method, using Apoptag fluorescein direct in situ apoptosis detection kits (ApopTag; Intergen, New York, NY, USA) according to the manufacturer's pro- 

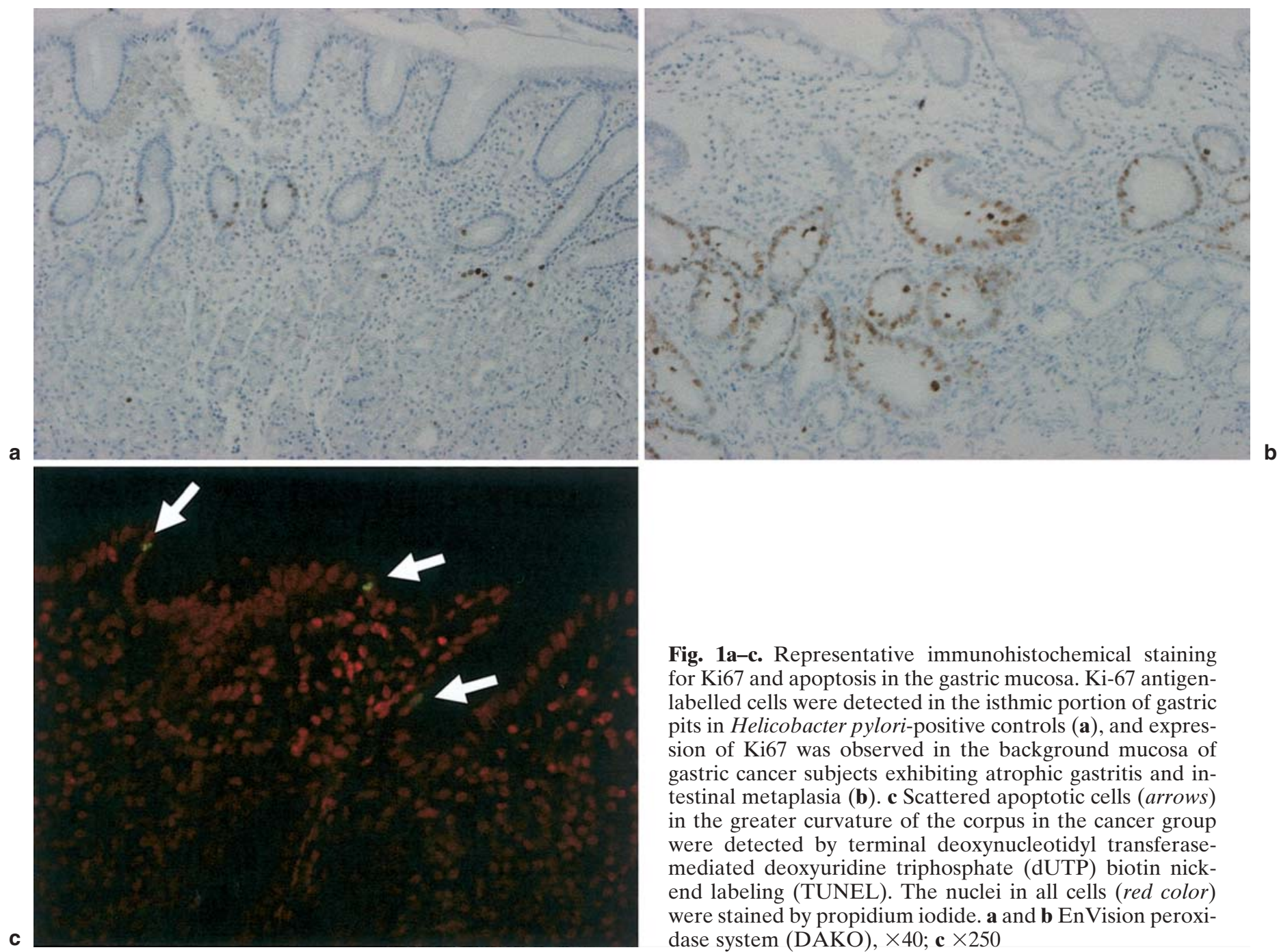

Fig. 1a-c. Representative immunohistochemical staining for Ki67 and apoptosis in the gastric mucosa. Ki-67 antigenlabelled cells were detected in the isthmic portion of gastric pits in Helicobacter pylori-positive controls (a), and expression of Ki67 was observed in the background mucosa of gastric cancer subjects exhibiting atrophic gastritis and intestinal metaplasia (b). c Scattered apoptotic cells (arrows) in the greater curvature of the corpus in the cancer group were detected by terminal deoxynucleotidyl transferasemediated deoxyuridine triphosphate (dUTP) biotin nickend labeling (TUNEL). The nuclei in all cells (red color) were stained by propidium iodide. $\mathbf{a}$ and $\mathbf{b}$ EnVision peroxidase system $(\mathrm{DAKO}), \times 40 ; \mathbf{c} \times 250$

tocol. The nuclei in all cells (red color shown in Fig. 1c) were stained by $0.25 \mathrm{mg} / \mathrm{ml}$ propidium iodide. Five hundred gastric epithelial cells in the foveolar region of longitudinally sectioned gastric pits were counted. The apoptotic index (AI) was defined as the ratio of TUNEL-positive cells (green color) to the total number of nuclei (red color), multiplied by 100 (Fig. 1c).

\section{Statistical analysis}

Values were expressed as medians and ranges. Mantel- $\chi^{2}$ analyses and the nonparametric MannWhitney $U$-test were performed to measure differences in demographic and clinical characteristics. The Kruskal-Wallis test was used for comparisons among groups, and statistical analysis for significant differences between two groups was performed using the nonparametric Mann-Whitney $U$-test. Linear correlations between parameters were performed by Spearman correlation analysis. A two-sided $P$ value of less than 0.05 was considered statistically significant. All statistical computations were performed using SPSS for Windows 11.0 (SPSS; Chicago, IL, USA).

\section{Results}

Initially 60 subjects were enrolled in the cancer group and 60 sex- and age-matched subjects were enrolled in the control group. However, 3 subjects in the cancer group were excluded because of a history of eradication therapy, and an additional 20 subjects ( 12 from the cancer group, 8 from controls) were excluded because of biopsy sample errors. The study groups thus consisted of 45 H. pylori-positive patients with EMR for early gastric cancer and $52 \mathrm{H}$. pylori-positive controls. Demographic and clinical characteristics of the study groups are shown in Table 1. Incomplete IM was present in 36 patients 
(80\%) in the cancer group and in $9(17.3 \%)$ of the $H$. pylori-positive controls. More patients in the gastric cancer group belonged to the incomplete IM group, and this difference between the two groups was highly significant $(P<0.001)$. Gastric cancer was associated with a higher frequency of incomplete IM (odds ratio [OR], 19.1; 95\% confidence interval [CI], 6.9-53.2; $P<0.001$ ). Malignant lesions were located in the antrum in 24 patients, and 15 patients had lesions in the corpus or angularis. In the remaining 6 patients, malignant lesions were found in both locations. No malignant lesion was located in the greater curvature of the corpus.

The scores for inflammation in the greater curvature of the corpus $(P=0.03)$, the scores for atrophy in the lesser curvature of the corpus $(P=0.001)$, and the scores for IM in each part of the stomach were significantly higher in the cancer group than in the control group. The scores for $H$. pylori in the antrum $(P=$ $0.002)$ and in the lesser curvature of the corpus $(P=$ $0.007)$ were lower in the cancer group than in the control group (Table 2).

The PI in each part of the stomach $(P<0.001$ in the lesser curvature of the corpus) and the $\mathrm{AI}$ in the greater curvature of the corpus $(P=0.02)$ were significantly higher in patients with gastric cancer than these values in the controls (Fig. 2). The median PI in the antrum was significantly higher (17.6 vs $12.6 ; P=0.009)$ in the incomplete-type IM group than that in the complete-

Table 1. Patient demographic and clinical characteristics

\begin{tabular}{lcc}
\hline & $\begin{array}{c}\text { Controls } \\
(n=52)\end{array}$ & $\begin{array}{c}\text { Patients with } \\
\text { cancer }(n=45)\end{array}$ \\
\hline Age, in years; mean (SE) & $70(1)$ & $68(1)$ \\
Sex; male/female & $42 / 10$ & $35 / 10$ \\
Current smokers & $25.0 \%$ & $18.2 \%$ \\
Regular alcohol intake & $40.4 \%$ & $46.7 \%$ \\
Type of intestinal metaplasia; & $19 / 24 / 9$ & $2 / 7 / 36$ \\
$\quad$ non/complete/incomplete* & & \\
$* P<0.001$ by Pearson $\chi^{2}$ test & & \\
\hline
\end{tabular}

$* P<0.001$ by Pearson $\chi^{2}$ test type IM group (Fig. 3). However, the AI was not different between these two groups.

The PI in the greater curvature of the corpus was significantly correlated with the score for activity $(r=$ $0.47 ; P<0.001)$ and the PI values in each part were correlated with the scores for atrophy and IM (IM, $r=$ $0.52 ; P<0.001$ in the lesser curvature of the corpus; Fig. $4 a)$. The AI was correlated with the score for activity $(r$ $=0.36 ; P<0.001)$ in the greater curvature of the corpus (Fig. 4b; Table 3).

The median scores for $H$. pylori in the greater curvature of the corpus were significantly higher (1.0 vs $0.5 ; P$ $=0.006)$ in patients with malignant lesions located in the antrum than the scores in patients with lesions in the corpus or angularis. The median scores for inflammation ( 2.5 vs $2.0 ; P=0.06)$ and PI (11.0 vs $10.3 ; P=0.06)$ in the greater curvature of the corpus also tended to be higher in the antrum group. However, the scores for atrophy, IM, and AI were not different between the upper-stomach group and the antrum group.

\section{Discussion}

Gastric cancer is the end result of $H$. pylori-related mucosal inflammation. The extent and location of atrophy and IM along the lesser curvature from the cardia to the prepyloric zone identifies patients with the highest cancer risk. ${ }^{12}$ Initially, inflammation in the antrum forms a favorable environment for $H$. pylori, whereas the onset of atrophy and IM results in a less favorable environment and a reduction in the bacterial load ${ }^{13}$ that may result in complete loss of $H$. pylori and a reversion of H. pylori seropositivity to seronegativity. Therefore, we focused on only $H$. pylori-positive patients and excluded those in whom the amount of gastric damage had already led to an absence of active $H$. pylori infection. We believe that this allowed a more valid comparison of epithelial cell turnover in patients with or without gastric cancer.

Table 2. Comparison of scores for histological findings between the control group and the cancer group

\begin{tabular}{|c|c|c|c|c|c|c|c|c|c|c|c|c|c|c|c|}
\hline & \multicolumn{5}{|c|}{ Antrum } & \multicolumn{5}{|c|}{ CLC } & \multicolumn{5}{|c|}{ CGC } \\
\hline & $\mathrm{AC}$ & IF & AT & IM & HP & $\mathrm{AC}$ & IF & AT & IM & HP & $\mathrm{AC}$ & IF & AT & IM & HP \\
\hline \multicolumn{16}{|c|}{ Control group } \\
\hline Median & 1.5 & 2.0 & 1.0 & 0.0 & 1.0 & 1.5 & 2.0 & 2.0 & 1.0 & 1.0 & 1.25 & 2.0 & 0.5 & 0 & 1.0 \\
\hline Range & $0-3$ & $0-3$ & $0-2.5$ & $0-2.5$ & $0-3$ & $0-3$ & $0-3$ & $0-3$ & $0-3$ & $0-3$ & $0-3$ & $0-3$ & $0-3$ & $0-3$ & $0-3$ \\
\hline \multicolumn{16}{|c|}{ Cancer group } \\
\hline Median & 1.0 & 2.0 & 1.25 & 1.0 & 0.5 & 1.0 & 1.5 & 2.5 & 2.0 & 0.5 & 2.0 & 2.5 & 1.0 & 1.0 & 1.0 \\
\hline Range & $0-3$ & $0-3$ & $0-3$ & $0-3$ & $0-3$ & $0-3$ & $0-3$ & $0-3$ & $0-3$ & $0-3$ & $0-3$ & $0-3$ & $0-3$ & $0-3$ & $0-3$ \\
\hline$P$ value & 0.56 & 0.48 & 0.08 & 0.001 & 0.002 & 0.87 & 0.81 & 0.001 & $<0.001$ & 0.007 & 0.06 & 0.03 & 0.39 & $<0.001$ & 0.06 \\
\hline
\end{tabular}

$P$ values by Mann-Whitney $U$-test

CLC, lesser curvature of corpus; CGC, greater curvature of corpus; AC, activity; IF, inflammation; AT, atrophy; IM, intestinal metaplasia; HP, Helicobacter pylori 


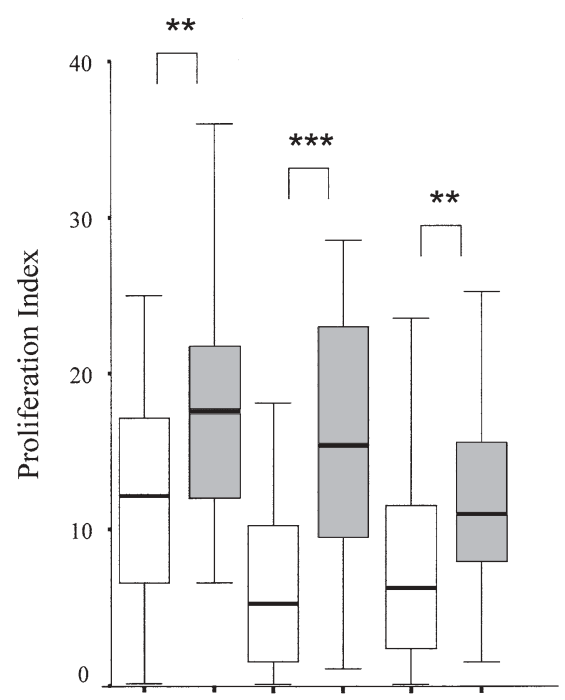

AT CLC CGC

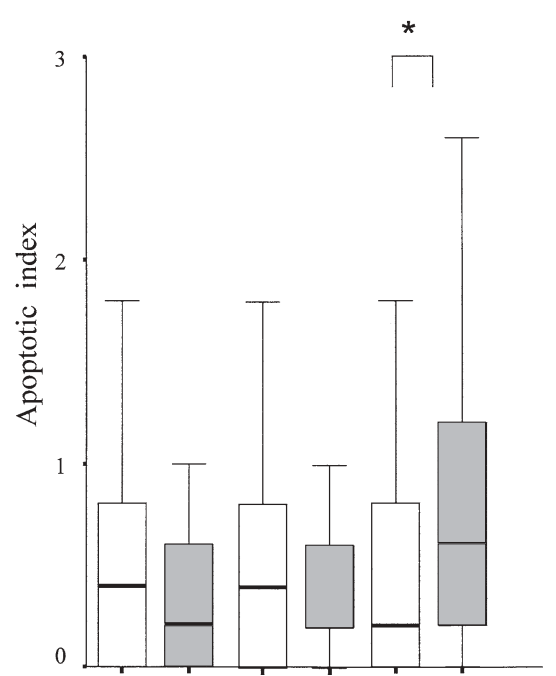

AT CLC CGC
Fig. 2. The proliferation index and apoptotic index in patients with gastric cancer and controls. The ends of the boxes define the 25th and 75th percentiles, with the bold lines showing the median values, and error bars defining the 10 th and 90th percentiles. $* P<0.05$; $* * P<0.01$; *** $P<0.001$ by nonparametric Mann-Whitney $U$-test. $A T$, antrum; $C L C$, lesser curvature of corpus; $C G C$, greater curvature of corpus. White bars, controls; gray bars, patients with cancer

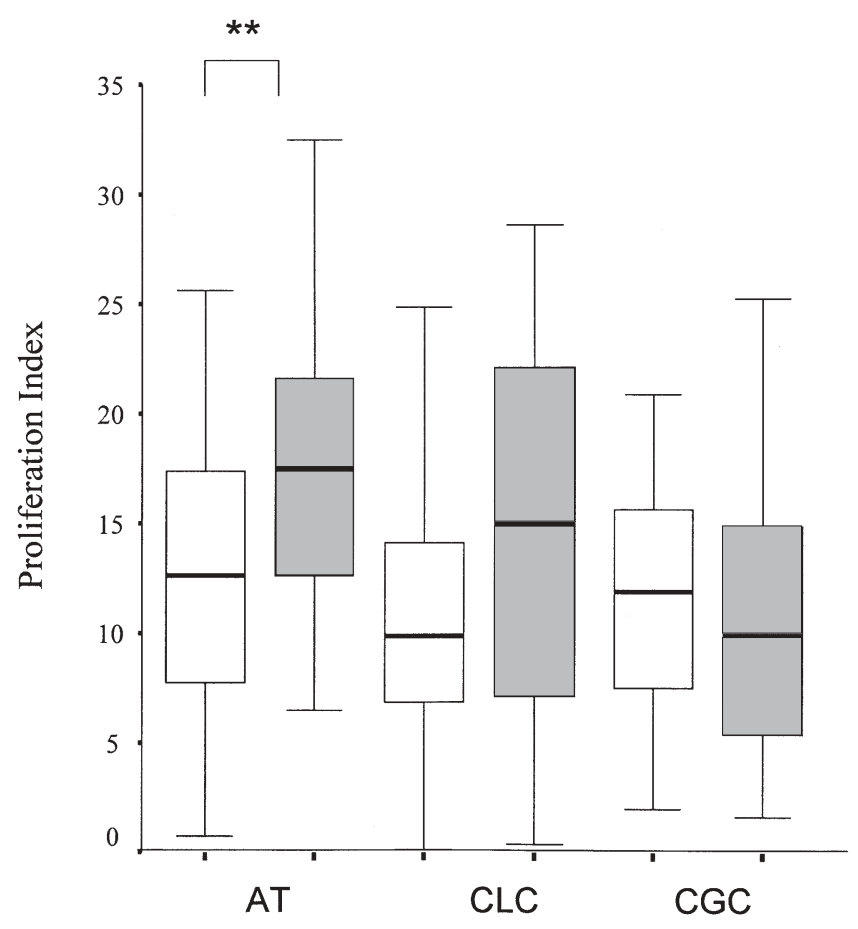

Fig. 3. The proliferation index in patients with complete intestinal metaplasia (white bars) and incomplete intestinal metaplasia (gray bars). The ends of the boxes define the 25 th and 75 th percentiles, with the bold lines showing the median values, and error bars defining the 10th and 90th percentiles. $* * P<0.01$ by nonparametric Mann-Whitney $U$-test. $A T$, antrum; $C L C$, lesser curvature of corpus; $C G C$, greater curvature of corpus
Although H. pylori infection is known to be associated with increased gastric epithelial cell proliferation, induction of apoptosis, and inhibition of DNA synthesis, ${ }^{7}$ the overall findings of its effect cell kinetics have been conflicting. ${ }^{14}$ This may be due, in part, to differences in the infecting strains of $H$. pylori or to the different clinical presentations which are associated with different patterns of gastritis. For example, it has been suggested that patients infected by $H$. pylori cagA- or babA2-positive strains have increased proliferation among gastric cells compared to patients infected by cagA- or babA2-negative H. pylori. ${ }^{15,16}$

Another major reason for different conclusions regarding the effects of $H$. pylori on cell kinetics may be related to the underlying histologic pattern. Some previous studies had reported that cell proliferation and apoptosis were correlated with inflammation. ${ }^{16-19}$ However, in the present study, the PI was most significantly associated with IM rather than with inflammation in the lesser curvature of the corpus. The PI and the IM score in each part of the stomach were significantly higher in patients with gastric cancer than these values in the controls. In contrast, the AI and scores for inflammation only in the greater curvature of corpus were significantly higher in the cancer group than in the controls, while the AI in the other parts tended to be lower in the cancer group. IM and inflammation accelerate cell proliferation; however, only inflammation induces apoptosis. Moreover, the treated gastric tumors in our patients had not existed in the greater curvature of the corpus (where the PI was relatively low and the AI was relatively high compared with PI and AI at other sites). 


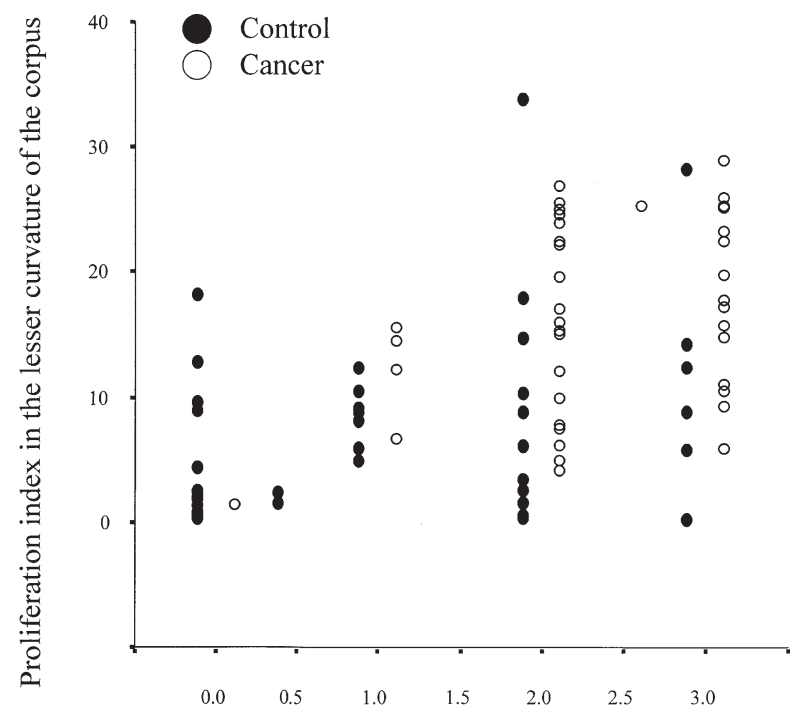

a

Score of intestinal metaplasia in the lesser curvature of the corpus

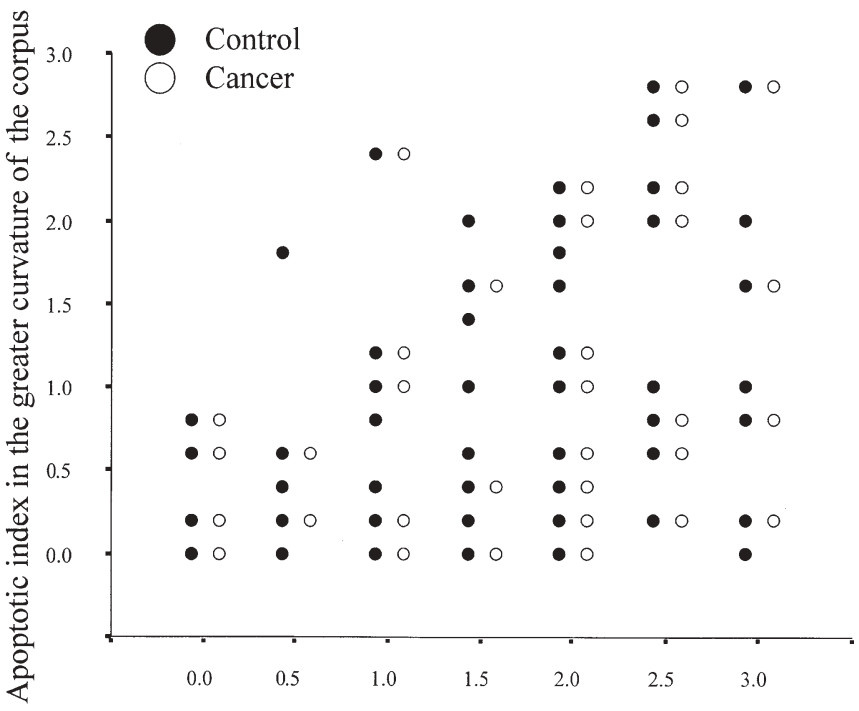

Score of activity in the greater curvature of the corpus

Fig. 4. a Correlation between the proliferation index and the score of intestinal metaplasia in the lesser curvature of the corpus. b Correlation between the apoptotic index and the score of activity in the greater curvature of the corpus

Table 3. Correlations between the scores for histological parameters

\begin{tabular}{llllllr}
\hline & \multicolumn{1}{c}{ AC } & IF & Atrophy & IM & HP & PI \\
\hline PI & & & & & & \\
AT & 0.05 & 0.05 & 0.19 & $0.32^{* *}$ & $-0.24^{*}$ & \\
CLC & 0.07 & 0.06 & $0.43^{* * *}$ & $0.52^{* * *}$ & -0.19 & \\
CGC & $0.47^{* * *}$ & $0.40^{* * *}$ & 0.18 & $0.28^{* *}$ & $0.36^{* *}$ & \\
AI & & & & & & \\
AT & $0.26^{*}$ & 0.09 & 0.13 & 0.05 & 0.02 & -0.10 \\
CLC & $0.23^{*}$ & $0.25^{*}$ & 0.02 & 0.10 & $0.21^{*}$ & 0.01 \\
CGC & $0.36^{* * *}$ & $0.30^{* *}$ & 0.17 & -0.05 & 0.06 & 0.27 \\
\hline
\end{tabular}

$P$ values by Spearman correlation analysis; $* P<0.05 ; * * P<0.01 ; * * * P<0.001$

AT, antrum; CLC, lesser curvature of corpus; CGC, greater curvature of corpus; AC, activity; IF, inflammation; IM, intestinal metaplasia; HP, Helicobacter pylori; PI, proliferation index; AI, apoptotic index

IM seems to be one of the most important factors affecting gastric mucosal cell proliferation, and this condition leads to proliferation-dominant cell kinetics, which may one of the components contributing to carcinogenesis.

Other recent studies have suggested that the development of IM is associated with the impaired regulation of gastric epithelial proliferation, which is not reversible following $H$. pylori eradication in areas where the oncoprotein p21ras is expressed. ${ }^{20,21}$ Apoptosis associated with $H$. pylori is also associated with increased epithelial proliferation, whereas, in areas of IM or dysplasia, proliferation remains increased but apoptosis reverts to normal levels following $H$. pylori eradication. ${ }^{5,22-25}$

Gastric IM is categorized into three types: I (complete), and II and III (incomplete). Type III IM typically shows the greatest genetic changes. Moreover, patients with type III IM are likely to be at a higher risk of gastric cancer. ${ }^{26}$ Thus, type III IM may be implicated in the severe imbalance of proliferation and apoptosis in the precancerous lesions. ${ }^{27}$ In the present study, more patients in the gastric cancer group compared to the control group belonged to the incomplete IM group, and the PI in the antrum was significantly higher in the incomplete IM group than in the complete IM group. Although an endoscopic follow-up study of patients with IM has reported the disappearance of IM, this result may be due to sampling error, as neither the presence nor the type of IM were consistent when endoscopic biopsies were repeated in the same regions of the stomach. ${ }^{28}$ However, when IM is extensive in the corpus, it is likely to be identified in biopsy materials, such 
that the finding of IM in the corpus is a marker of corpus atrophy.

There are two other studies comparing epithelial cell turnover in patients with and without gastric cancer, reporting conflicting results on cell kinetics. ${ }^{29,30}$ One study $^{29}$ reported that the AI in the corpus, which correlates with the grade of glandular atrophy, was significantly higher in patients with intestinal-type cancer compared with controls. The PI was not significantly different between these groups. ${ }^{29}$ However, the number of their cancer subjects was small $(n=32)$ and may be inadequate for statistical purposes. The other study ${ }^{30}$ reported that the PI in the greater curvature of the corpus and the lesser curvature of the antrum was higher in 63 cancer patients than in $97 \mathrm{H}$. pylori-positive nonulcer dyspepsia subjects. The AI in the cancer group was lower at all sites, including the greater curvature of corpus. ${ }^{30}$ One possible reason for the difference between this study's findings, ${ }^{30}$ and our results could be the discrepancy in ages between their cancer and control groups. Moreover, in our study, only early and intestinal types of gastric cancer were evaluated. Histologically, gastric cancers have been classified into two main histological types, diffuse and intestinal, which have differing characteristics and possibly differing pathogenesis. Diffuse-type gastric cancer arises independently of IM, and the AI in cancer lesions is significantly higher in intestinal-type than in diffuse carcinoma. However, the PI is higher in diffuse compared to intestinal tumors. ${ }^{31}$

In patients with malignant lesions located in the antrum, the scores for H. pylori were much higher than the scores for inflammation, and the PI tended to be higher in the greater curvature of corpus, where the scores for atrophy and IM were not different compared with those in the upper stomach. The gastritis in the greater curvature of the corpus induced by $H$. pylori is probably associated with the development of gastric cancer, especially in the antrum, and there may be site-specific differences characteristic and possibly pathogenetic of gastric cancer. A larger-scale investigation to study the cancer phenotype is needed to confirm site-specificity.

In patients with gastric cancer, severe inflammation still exists in the greater curvature of the corpus, accelerating the gastric mucosal cell turnover. However, at other sites, especially the lesser curvature of the corpus, where severe glandular atrophy has developed, IM occurs, accompanied by a reduction of $H$. pylori density, resulting in the same type of imbalance between cell apoptosis and proliferation as that seen in neoplasia. Therefore, in conclusion, it appears that H. pyloriinduced gastritis is associated with increased cell proliferation and apoptosis, and likely results in atrophy and IM, leading to gastric cancer. IM seems to be one of the most important factors affecting cell proliferation, and may be components of carcinogenesis that results in proliferation-dominant cell kinetics.

Acknowledgments. This work was supported in part by US Public Health Service Grant R01DK61410 to J.L.M.

\section{References}

1. Correa P. Chronic gastritis as a cancer precursor. Scand J Gastroenterol Suppl 1984;104:131-6.

2. Correa P, Haenszel W, Cuello C, Zavala D, Fontham E, Zarama $\mathrm{G}$, et al. Gastric precancerous process in a high risk population: cohort follow-up. Cancer Res 1990;50:4737-40.

3. Correa P. Helicobacter pylori and gastric carcinogenesis. Am J Surg Pathol 1995;19(Suppl 1):S37-43.

4. El-Zimaity HM, Ota H, Graham DY, Akamatsu T, Katsuyama T. Patterns of gastric atrophy in intestinal type gastric carcinoma. Cancer 2002;94:1428-36.

5. Xia HH, Talley NJ. Apoptosis in gastric epithelium induced by Helicobacter pylori infection: implications in gastric carcinogenesis. Am J Gastroenterol 2001;96:16-26.

6. Konturek PC, Bielanski W, Konturek SJ, Hahn EG. Helicobacter pylori associated gastric pathology. J Physiol Pharmacol 1999;50: 696-710.

7. Wagner S, Beil W, Westermann J, Logan RP, Bock CT, Trautwein C, et al. Regulation of gastric epithelial cell growth by Helicobacter pylori: evidence for a major role of apoptosis. Gastroenterology 1997;113:1836-47.

8. Jones NL, Shannon PT, Cutz E, Yeger H, Sherman PM. Increase in proliferation and apoptosis of gastric epithelial cells early in the natural history of Helicobacter pylori infection. Am J Pathol 1997;151:1695-703.

9. Moss SF, Sordillo EM, Abdalla AM, Makarov V, Hanzely Z, Perez-Perez GI, et al. Increased gastric epithelial cell apoptosis associated with colonization with cagA + Helicobacter pylori strains. Cancer Res 2001;61:1406-11.

10. Peek RM Jr, Moss SF, Tham KT, Perez-Perez GI, Wang S, Miller GG, et al. Helicobacter pylori cagA + strains and dissociation of gastric epithelial cell proliferation from apoptosis. J Natl Cancer Inst 1997;89:863-8.

11. Dixon MF, Genta RM, Yardley JH, Correa P. Classification and grading of gastritis. The updated Sydney System. International Workshop on the Histopathology of Gastritis, Houston 1994. Am J Surg Pathol 1996;20:1161-81.

12. Cassaro M, Rugge M, Gutierrez O, Leandro G, Graham DY, Genta RM. Topographic patterns of intestinal metaplasia and gastric cancer. Am J Gastroenterol 2000;95:1431-8.

13. Karttunen T, Niemela S, Lehtola J. Helicobacter pylori in dyspeptic patients: quantitative association with severity of gastritis, intragastric $\mathrm{pH}$, and serum gastrin concentration. Scand J Gastroenterol Suppl 1991;186:124-34.

14. Cahill RJ, Kilgallen C, Beattie S, Hamilton H, O'Morain C. Gastric epithelial cell kinetics in the progression from normal mucosa to gastric carcinoma. Gut 1996;38:177-81.

15. Rokkas T, Ladas S, Liatsos C, Petridou E, Papatheodorou G, Theocharis S, et al. Relationship of Helicobacter pylori CagA status to gastric cell proliferation and apoptosis. Dig Dis Sci 1999;44:487-93.

16. Yu J, Leung WK, Go MY, Chan MC, To KF, Ng EK, et al. Relationship between Helicobacter pylori babA2 status with gastric epithelial cell turnover and premalignant gastric lesions. Gut 2002;51:480-4.

17. Baldini L, Pretolani S, Bonvicini F, Miglio F, Epifanio G, Gentiloni Silveri N, et al. Effect of Helicobacter pylori infection, age and epithelial cell turnover in a general population at high risk for gastric cancer. Panminerva Med 1999;41:187-92. 
18. Lynch DA, Mapstone NP, Clarke AM, Jackson P, Moayyedi P, Dixon MF, et al. Correlation between epithelial cell proliferation and histological grading in gastric mucosa. J Clin Pathol 1999; $52: 367-71$.

19. Sougioultzis S, Foukas PG, Tzivras M, Kourtessas D, Gorgoulis VG, Davaris $\mathrm{P}$, et al. Alterations in the proliferating compartment of gastric mucosa during Helicobacter pylori infection: the putative role of epithelial cells expressing p27(kip1). Mod Pathol 2003;16:1076-85.

20. Polat A, Cinel L, Dusmez D, Aydin O, Egilmez R. Expression of cell-cycle related proteins in Helicobacter pylori gastritis and association with gastric carcinoma. Neoplasma 2002;49:95100.

21. Ierardi E, Francavilla R, Panella C. Effect of Helicobacter pylori eradication on intestinal metaplasia and gastric epithelium proliferation. Ital J Gastroenterol Hepatol 1997;29:4705.

22. Scotiniotis IA, Rokkas T, Furth EE, Rigas B, Shiff SJ. Altered gastric epithelial cell kinetics in Helicobacter pylori-associated intestinal metaplasia: implications for gastric carcinogenesis. Int J Cancer 2000;85:192-200.

23. Unger Z, Molnar B, Szaleczky E, Torgyekes E, Muller F, Zagoni $\mathrm{T}$, et al. Effect of Helicobacter pylori infection and eradication on gastric epithelial cell proliferation and apoptosis. J Physiol (Paris) 2001:95:355-60.

24. Zhang Z, Yuan Y, Gao H, Dong M, Wang L, Gong YH. Apoptosis, proliferation and $p 53$ gene expression of $\mathrm{H}$. pylori- associated gastric epithelial lesions. World J Gastroenterol 2001; 7:779-82.

25. Ebert MP, Schandl L, Malfertheiner P. Helicobacter pylori infection and molecular changes in gastric carcinogenesis. J Gastroenterol 2002:37(Suppl 13):45-9.

26. Tahara E. Molecular mechanism of human stomach carcinogenesis implicated in Helicobacter pylori infection. Exp Toxicol Pathol 1998;50:375-8.

27. Filipe MI, Mendes R, Lane DP, Morris RW. Assessment of proliferating cell nuclear antigen expression in precursor stages of gastric carcinoma using the PC10 antibody to PCNA. Histopathology 1993;22:349-54.

28. El-Zimaity HM, Ramchatesingh J, Saeed MA, Graham DY. Gastric intestinal metaplasia: subtypes and natural history. J Clin Pathol 2001;54:679-83.

29. Yoshimura T, Shimoyama T, Tanaka M, Sasaki Y, Fukuda S, Munakata A. Gastric mucosal inflammation and epithelial cell turnover are associated with gastric cancer in patients with Helicobacter pylori infection. J Clin Pathol 2000;53:532-6.

30. Wambura C, Aoyama N, Shirasaka D, Sakai T, Ikemura T, Sakashita M, et al. Effect of Helicobacter pylori-induced cyclooxygenase-2 on gastric epithelial cell kinetics: implication for gastric carcinogenesis. Helicobacter 2002;7:129-38.

31. Ikeda M, Shomori K, Endo K, Makino T, Matsuura T, Ito H. Frequent occurrence of apoptosis is an early event in the oncogenesis of human gastric carcinoma. Virchows Archiv 1998; 432:43-7. 